learning, if contrasted with residents in the more boreal regions, who feel ever anxious to obtain knowledge from any source, whether foreign or domestic. The former are, to quote the saying of an illnatured critical foreigner, oftener teachers than scholars of other nations, according to their own estimation; and usually believe themselves best entitled to lead the van, instead of following in the wake of modern civilization.

\title{
REvIEW XIII.
}

\section{A System of Surgery, Theoretical and Practical; in Treatises by} various Authors. Edited by T. Holmes, M.A. Cantab., AssistantSurgeon to the Hospital for Sick Children. In Four Volumes. Vol. I., pp. 850.-London, 1860.

"A TENDENCY to greater exactness of thought in questions of cause and effect," it is remarked by one of the writers in the volume before us, "has been part of the general scientific development of our time -belonging not exclusively to medicine, but perhaps rather coming to medicine by contagion from physical and chemical studies. Fortunately, however, it has come to us." The appearance of a new work, long wanted, which is to comprise the whole range of surgical practice as understood in the present day in England, naturally leads us to inquire how it happens that such a work is new, and how it is that the want of it is only now to be supplied. It had long been felt that however high the standard of surgery had been raised in this country, it was not to the credit of the professors and practitioners of the science, that there was no modern work sufficiently comprehensive in its pages to compare with the productions of other countries, which at once gave a view of the whole range of the science and practice of surgery, and was at the same time the production of individual research and experience, rather than a compilation from the labours of other men. Contented to take their stand on ground they knew they could hold, it seemed as if the leaders and the teachers in the English schools of surgery were to be known by their well-earned title to safe and successful practical knowledge-the pride, as it is thought, of the practical Englishman; rather than by any claims they could put forward to higher honours gained by toil in the more laborious fields of original investigation and research. At all events, there was no single work, as a written record of the results of such labours, by which their high claim to pre-eminence could be tested, or from which it could be proved. Where the possession of such a title was not known, it could safely be called in question; and the high claims of the English schools could be challenged by others who, perhaps, looked around them from a lower standing on the slopes of the surgical Olympus.

The projectors of the work, of which one volume is now before the profession, have undertaken to supply the acknowledged deficiency; and for this purpose they have formed into a "system" a collection of 
essays or treatises by different writers on the numerous subjects comprised in the general term of "Surgery." The difficulty of obtaining qualified contributors to unite in the general plan of a work which should be a complete expression of the opinions and experience of many men; and, still more, to act in concert in the execution of it, and to perform with punctuality their engagements with their common editor and the professional public, has been one chief obstacle in the way of the completion of former undertakings of a similar comprehensive nature, though the hope of it has often been conceived by active minds.

The essays in the present volume, which are professedly limited to subjects of general pathology, are for the most part introductory to the fuller treatises which are to appear in subsequent volumes.

The opening essay is by Mr. Simon, who takes for his subject the pathology and treatment of Inflammation generally; a subject, he says, "of large scope and of equally large importance; the study of which seems to branch almost throughout the whole subject matter of surgery :" and he adds, "assuredly it is no exaggeration to say that rational surgery depends more upon a knowledge of the inflammatory process, than upon all other pathological knowledge put together." The inflammatory process, in its most characteristic type, is illustrated by the history of the rise of a carbuncle, and its progress, through the separation of the slough, and the healing, with the formation of a sound cicatrix. Mr. Simon, we see, calls it a slough, and minutely describes the microscopic characters which entitle him to declare it to be so. Hyperæmia invariably attends inflammation, and is essential to the inflammatory process, whether that be a destructive or a productive process; and it is seen in all forms of increased nutritive activity. It can go on for months without producing textural change, without causing a single pus-cell to grow, or a single texturegerm to die. A part is not inflamed because it contains more blood; it receives more blood because it is inflamed.

Our knowledge, such as it is, of the state of the capillary circulation in inflamed parts, is summed up by Mr. Simon in these conclusions:

"That within the area of stasis, the blood has lost the fluid in which its corpuscles should float; that the circulation of the corpuscles is delayed; that they collect against the wall of the capillary, as though by mutual cohesiveness, though they do not, when removed, seem more cohesive than in blood of healthy parts; that there is increased infiltration of the contiguous textures, and that stasis occurs with more or less facility in proportion as the liquor sanguinis is more or less transudable, and it does not result from alteration in calibre in the vessels of the part; that the cause of its production is an influence excited on the blood by textures within the area of stasis; that this influence is, mechanically speaking, of a suctional kind, which differs but in degree from that which the textures naturally exercise on the blood as it passes amid them."

The symptoms and causes of inflammation, as taught and described by former writers, much of whose doctrines may still be accepted as true, are traced with the closeness of investigation and clearness and comprehensiveness of arrangement which have distinguished all $\mathbf{M r}$. 
Simon's former writings. The phenomena of inflammation are modified phenomena of textural life; the power which produces them is the power which produces the ordinary phenomena of textural life, and fully to explain inflammation would, in fact, be to explain life. Mr. Simon closes a valuable and instructive essay with a brief history of the literature of the subject; "the special study of which," he writes, "without undue partiality, an Englishman may be glad to say dates from the labours of John Hunter."

Mr. Coote contributes two short essays-one of them on Abscess. $\mathrm{He}$ gives in clear language, and in very few sentences, the most approved modern doctrine of the origin of pus : in behalf of which it must be said, as of much other learning that we labour to accumulate, that it seems a weak part of the doctrine that a writer is forced to con-fess that some of what is most necessary to the support of it "has yet to be proved." The possibility of the absorption of pus, though now known to be of frequent occurrence, rather than an occasional and very rare exception to a pathological law, is not yet universally admitted, if we may judge by the practice, still followed by some surgeons, of incising every fluctuating swelling. Where there is room for a doubt, there will generally be found some ground to support it; and of a soft and fluctuating swelling, which has been allowed to disappear without active surgical interference, it will many times be a question after all, which can never be solved, whether it was indeed pus.

The rules laid down for opening abscesses are liable, we think, to some difference in opinion. The bleeding lancet, if made, as lancets are now usually made for the pocket-case, sufficiently strong in the blade not to bend, and strong enough in the point not to break, we have generally found the most satisfactory instrument for opening the abscesses under the common integument; and these are the abscesses for which an opening is most frequently needed, or, perhaps we should say, is most frequently made. It is a less formidable instrument to produce in the sick-room, and we think it may be commended as doing the work sufficiently well. For chronic suppuration, for the abscess under the deep fascia, "a thin, yet broad-shouldered, sharp-cutting, dotible-edged knife, or scalpel," recommended by Mr. Coote, will be found more serviceable-greatly superior, according to our observation, to the curved cutting instrument, the bistoury, or the old-fashioned "abscess lancet." The rapid division of inflamed tissues, such as those generally lying over an abscess, of the integument especially, is attended with severe pain ; and Mr. Coote's "rapid and bold plunge" of the instrument will often be found to give unnecessary pain in the beginning of an operation, at the end of which he justly condemns all probing and squeezing, as "a proceeding extremely painful" to the patient. The poultice, after the first application, may well be dispensed with; for it rather tends to keep up the suppuration, and by weakening the vessels, so far interferes with the recommendation to "leave the remainder of the cure to Nature." In connexion with several cases well related, Mr. Coote describes the different regions of the body in which abscesses are found sufficiently often to become sub. 
jects of surgical interference, or, when of rare occurrence, to give rise to difficulty in diagnosis.

$\mathrm{Mr}$. Paget is one of the largest contributors to the present volume ; four separate articles are from his pen. After describing the structure and anatomical characters of Fistula and Sinuses, as commonly found in practice, Mr. Paget proceeds to consider their different modes of formation, with their general pathology. The many different methods of treatment, constitutional, local, and operative, are passed in review, with concise and practical remarks on their value as applicable to the different diseases, and as affording meaus of cure when stationary under the unaided efforts of Nature: for these, unhappily for the patient, are not among the diseases in which Nature alone is wont to show the most brilliant success.

Under the head of Gangrene, Mr. Coote includes the different terms which have been applied to mortification, or "the doing to death," of different textures of the body, internal or extermal ; "but these different terms," he adds, " have been used loosely, and it would lead to no practical result to attempt any very strict definition." Ulceration and mortification are often mingled ; the ulcerative and sloughing processes may go on, as it were, hand in hand. Still, surgically speaking, there is a wide difference between ulceration and gangrene, beyond the extent of the part involved in the spreading destructive disease, and the more intimate sympathy of the constitution with one than in the other; however difficult it may be to frame a definition which shall separate them by a broad line. The causes of gangrene are numerous; the attempts to interpret them aright have been conflicting, and the interpretation not always satisfactory. The death of an inflamed part, it has been remarked, is a very complex matter. The rules for the treatment of mortification, $\mathrm{Mr}$. Coote says, are much simpler than in times gone by ; but the means we possess of controlling it are few in number.

Mr. Coote remarks that surgeons do not often witness death from the unchecked progress of traumatic gangrene, in a part destroyed by direct local violence. The fearful amount of local injury, of which it is thought that gangrene will be the certain termination, is too clear an indication for the immediate removal of the injured member, and it is seldom indeed-very seldom, we should say-that the patient is not himself sensible of the urgent necessity for an operation, or that he refuses, when it is really required, to be guided by the judgment of the surgeon when he advises immediate amputation. Yet we do find now and then that he refuses; and we do find sometimes, and when we have hardly expected it, that gangrene in such almost hopeless circumstances will stop short of the extinction of life; as we also find that the amputation of a limb, even at a distance from the injury, and far out of reach, as one would think, of all contaminating influences, does not always save life. The cases in which, in the absence of constitutional causes, predisposing or exciting, the gangrene is most frequently seen to run its course with rapidity, are those where the injury has been to a large nerve or primary bloodvessel, and for which, upon its 
occurrence, the indication for amputation was not sufficiently distinct. The secondary mortification following injury less severe in its character is less rapid in progress, and more frequently stops, or performs a spontaneous amputation.

Mr. Coote gives a full and faithful description of hospital gangrene, the "good example" of the disease which forms the subject of his essay. Constitutional symptoms, he correctly says, may be generally observed; and where the disease attacks large wounds, such as stumps after amputation, it will often seem as if they were really the precursors of the local manifestation of the disease; though we suspect a more careful observation of the wound and its secretion would have shown early enough that there, at least, all was not right. As might be inferred in a state sometimes resulting from atmospheric contagion, or inoculation, the local condition often precedes the sympathy of the system days-aye, weeks. In the wards of the hospital where Mr. Coote has gained his chief experience of the disease, constitutional treatment, most carefully followed out, failed forty years ago to cure a single case of the disease, every one of which in its utmost fury was instantly and permanently checked by local treatment. Pure nitric acid was "generally preferred," then as now, for the reason that no known caustic has such an affinity for animal matter. If applied in its full strength, it is found to be less painful than when diluted. To some extent this is the case with other caustics, and applied in other cases. Indeed, we have heard it suggested that a strong caustic might not inaptly be called an anodyne.

The subject of Ulcers is treated by Mr. Paget-limiting his essay to the consideration of those forms of ulcer which appear in the integuments-as being of chief interest from the surgeon's point of view. It simplifies a work to have some mode of arrangement and classification for purposes of description; and $\mathrm{Mr}$. Paget describes each of the chief varieties of ulcers, of which sufficiently numerous instances are seen to allow of such arrangement, according to their origin and character; and when not of specific origin, they are named from some prominent or distinctive characteristic. Many of these ulcers, so indicated as inflammatory, eczematous, \&c., are also capable of classification as simple ulcers, without constitutional vice, to which accident has added some complication - as local inflammation, eczema, \&c. Among the patients presenting themselves at public institutions, such complications are often epidemic, if we may judge from the number of patients in whom they are seen. "The characters of ulcers," Mr. Paget remarks, "if more fully studied, both in wellmarked and in modified and complicate examples, would be found as various, and severally, at least, as well-defined, as are those of cutaneous eruptions; and with the better diagnosis that would be thus attained, there might follow a much better discrimination of the means of treatment appropriate for each."

An essay upon Erysipelas is the contribution by Mr. De Morgan. "The term," he observes, "has been applied to a variety of forms of inflammation which probably have no true connexion with one 
another." Of the two chief varieties now universally distinguished, simple and phlegmonous, Mr. De Morgan thinks that there is no real boundary between them. The various modifications of these he regards but as phases of one disease, which has its seat not merely in the part, but in the system: "The form which the disease may take is determined by the state of the system, the temperament, habits, \&c., of the individual, or the external causes which produce it." There are, we think, some prominent points of distinction to be noted between the two more commonly observed forms, which tend to make a boundary between them less open to question. If we may believe the practitioners beyond the limits of this great city, in the purer air of the country, or in less crowded towns, and in the county hospitals, phlegmonous erysipelas is rare, while simple erysipelas is of frequent occurrence. The phlegmonous form, if contagious, must be slightly so in comparison with the simple. The phlegmonous species is not often seen at hospitals in its early stages, at the period when local depletions or a milder treatment than free incision is likely to be beneficial and to arrest its progress. We think it may be open to question whether the phlegmonous form is not in reality an inflammation of the areolar and adipose tissues, of a specific character, spreading rapidly with gangrene, inwards to the fascia and muscles, and outwardly to the common integument, rather than a disease of common origin with the simple form-a disease " not of itself a formidable complaint."

Mr. De Morgan has condensed all that is valuable and practical in the literature of the subject; and in reviewing with him the opinions and practice of former writers, we are pleased to receive from his own pen the theory and practice of a surgeon so well able to give the result of his own large experience. Putting the fullest confidence in constitutional treatment, he gives in detail the many local applications recommended to relieve or cut short the attack; but he does not set a high value on them, for he has not found them realize fully the merits claimed for them. Indeed, it almost seems that the more nearly these approach to harmlessness, the greater is their merit as medicinal agents.

Mr. Callender contributes an essay on Pycemia, restricting his remarks to those cases in which the mischief begins in a diseased or injured part. The materies morbi he considers to be a modification of the blood, resulting from various predisposing causes: "Constituents are probably left in it, unused or unremoved, from defective secretion, or embarrassed excretion, some of which are ready, on the least disturbance, to undergo decomposition. Chemistry fails to give us conclusive evidence of these changes." The systemic infection is caused by some animal poison; and, influenced by predisposing causes, it becomes a general disease, more or less rapid in its progress, with symptoms allied to those of typhoïd fever, or it may be limited to a local action more or less diffused. Mr. Callender has noted with care the pathological changes, as deduced from examinations, for which the numerous fatal cases give abundant opportunities for observation; and 
he gives a selection from the views taken by such previous writers of authority as have received the greatest amount of credit, and he relates the arguments by which they have been supported. Although some cases have been recorded in which patients have recovered, Mr. Callender remarks that "pyæmia is dependent upon a state with which life can scarcely be prolonged;" and he urges most strongly, as a thing of the greatest importance, where therapeutics fail so signally, the paramount necessity of attending to prophylactic measures.

$\mathrm{Mr}$. Poland, in treating the subject of Tetanus, remarks that the degree of the local injury bears no relation to the severity of the symptoms; yet the relative proportions which the occurrence of the disease bears to the several surgical lesions show a great preponderance of cases among the more severe varieties of injury and accident, such as compound fractures, burns, and injuries to the fingers and toes. With the pathology "still involved in great obscurity," and in the absence of "an appropriate means of cure," Mr. Poland abstains from offering any remarks on the numerous medicines which have been administered and proposed as remedies. Woorara, one of the most recent, first used by an Italian surgeon, " has at present not fulfilled its purpose ;" no real success, we believe, followed the use of it upon the wounded after Solferino.

A short essay on Delirium Tremens, contributed by Dr. Barclay, contains a good medical description by a physician of the special characters of the disease in its most striking form. "The information derived from anatomical research," he remarks, "is worthy of study, because, as it tells how death is actually brought about, it offers suggestions as to how the fatal event may be prevented or warded off;", and he urges the importance of clinical study, as it alone furnishes data on which we can rely in forming any notion of the true nature of the disease, where anatomy often fails as a guide. "The moral management of the patient" is recommended as "the point of most importance in practice,"- coercive measures being justifiable only under unavoidable necessity. This necessity unfortunately too often exists in the circumstances in which the unhappy patient is placed. Where quiet can be secured, and complete separation from all continuance of excitement, as in the lunatic asylums (where cases often happen), personal restraint is not found to be necessary; the patient, if violent, is put into a small ward by himself, from which the light can be excluded, with padded floor and padded walls, where he cannot hurt himself; and it is found that he obtains rest, and the cure follows.

Mr. Savory contributes an essay on Scrofula, a word "in one respect at least unfortunate, because very different significations are attached to it;" he proposes to understand by it " a certain disease or defect of the constitution, in which there is a tendency to the production and deposit of tubercle (a substance which possesses no inherent power of growth) in various tissues and organs." In fact, he uses the term as generally identical with tuberculosis. He describes the minute structure of tubercle and the elements proper to it as recognisable under the microscope, and he traces the history of its transition through the 
various changes it undergoes. The symptoms of scrofula are the symptoms of debility; it is a disease of early life, common before the middle period, rare after it-the predisposition undoubtedly hereditary. "Can the diathesis, if not hereditary, be acquired?" is a question to which Mr. Savory thinks it is, from the nature of the subject, almost impossible to give an unequivocal answer.

A shorter essay follows, by the same hand, on Hysteria-a disease equally a disease of debility, seldom seen in healthy, or, at all events, not in strong constitutions - generally associated with some form of debility of the system or defect in the general health. Mr. Savory describes the symptoms of hysteria, whether pure or complicated, and the diseases which are mimicked by the patients under the influence of this obscure affection, and he points out that the symptoms are exaggerated, and the difficulty of diagnosis is incalculably increased. when hysteria supervenes upon real disease, - a combination which, however, is comparatively rare.

Mr. H. Lee contributes a treatise on Syphilis. His experience of venereal diseases has been gained by long observation of the natural progress of the diseases occupying the parts concerned in sexual congress. $\mathrm{He}$ is candid in his acknowledgments that much yet remains to be done for the complete elucidation of those cases which give rise, to such great contrariety of opinions and practice. In the introductory chapter he canvasses many of the opinions which have been of late years published by foreign practitioners upon the subject of inoculation with venereal virus, and the conclusions which have been drawn from them. Much of this experimental inoculation has been made with matter taken from various sores, not all probably of the same specific character, or in the same stage of progress. It is also an objection that the experiments have been for the most part, if not always, made upon the diseased subject. In order to show the natural progress of the original disease, if a constitutional affection, another subject should be taken, in good health-an expedient which it is not likely will ever be adopted extensively, - at least, in this country. Moreover, we think these experiments, even on the person of the patient, are not so free from danger as is generally supposed. We have known the ulcer produced by inoculation continue to spread, and proceed unchecked, till the death of the unfortunate patient put a period to farther observation. The scaly form of disease can perhaps be always introduced by inoculation from the secretion of the primary sore, and it may proceed on its regular course, through known stages, little (if at all) modified by peculiarity of constitution. Not so the phagedenic; this takes root less readily, unless when engrafted on a fit stock. At all events, we seldom see it, either hereditary or acquired, in its full characteristic virulence, except in what may be called rotten constitutions, or if in young and (what might be thought) healthy subjects, young rakes, fair people with thin skins and thin bloodvessels. As Mr. Lee says, "pustular eruptions do occur; but they are rare in unimpaired constitutions." It need not be a matter of surprise that some forms of known constitutional disease, or cachexia, 
cannot be engrafted by inoculation. Cancer, for instance, has never been ; and experiments have been abundant on the persons of surgeons engaged in operations. A professional friend once told us that he and others of his acquaintance had tried in vain to inoculate measles.

Mr. Lee's investigations confirm the observation of some former writers of the great numerical frequency of sores followed by irritation of the absorbents, and extensive suppurating buboes, without constitutional infection, or "secondary" symptoms; and also that there are constitutional affections, slow in progress, and not in general preceded by visible contamination of glands-the irritation and enlargement of the lymphatics often escaping the patient's observation, as, we think, the original sore of the scaly form will sometimes. His investigations also go to support the opinion long and publicly maintained, that there is a steadily progressive venereal disease, though of (pathologically speaking) a mild character, not tending to ulceration-a disease which it has been too much a fashion in the present day to doubt and even to deny. We think the distinction proposed between a "suppurating sore" and an "infecting chancre" is a good and practical one; and we shall be glad to see Mr. Lee distinguish further between the secondary affections. The "suppurating sores" require a further separation into those which are harmless and those which are followed by phagedenic or other secondary affections.

The proposal to cut short the progress of venereal diseases by excision of the primary sore does not find favour with Mr. Lee: "the cut surface will in a few days take on a specific action." Mr. Evans pointed out, many years ago, that the diseased action of the sore, "which," he says, " is more frequently met with than all other ulcerations on the parts of generation put together," extends in many instances a considerable distance beyond the seat of the sore, and that, if leeches be applied to the groin for the reduction of bubo, the wounds made by them often become sores of the same form. But, in truth, the patient generally applies too late for excision to give a fair chance of removal of the disease: the poison has taken effect by the time he discovers the sore which gives rise to his anxiety. We are not aware that the vaccine vesicle has ever been excised. Dr. Walker's experiment was the nearest approach to this, and he considered that the constitution was sufficiently infected, notwithstanding the strange proceeding.

Mr. Paget, acknowledging the great difficulty felt by most preceding writers of a correct definition of Innocent Tumours, arranges these irregular growths-these "lumps" that ought not to be-according to their "chief and most nearly constant distinctive characters," thus forming a classification which shall include all known forms of visible swellings in the body, or all that are of interest in practice, or (we may add, perhaps) that are of alarm to a patient; a "tumour," wherever situated, being generally a source of anxiety if its nature is doubtful. The modes of development and onward growth, with their minute anatomical and pathological structure, are given; the diffi- 
culties in many of them, met with in practice, and interfering with what have been thought established rules for diagnosis, are carefully considered; and many valuable remarks are made upon the surgical treatment of the different kinds of tumours, to which the reader may refer again and again with advantage.

Mr. Moore contributes a long and carefully drawn essay on Cancer. "The subject," he remarks, "is one in which there is the strangest disproportion between the amount and the practical value of our knowledge." The material of the essay, we may suppose, is the result of his own experience, confirmed by that of his colleagues, and gathered among the ample records of the Middlesex Hospital. The various forms in which cancer presents itself to the notice of the surgeon are arranged by Mr. Moore as Scirrhous, Medullary, Melanotic, Epithelial, and Osteoïd. Of these shades and shapes of malignant disease, scirrhus is the most frequent form in this country, and "in early stages of the disease it often possesses every character of a tumour but that of swelling." Mr. Moore calls attention to the very early period at which infection takes place in the absorbent glands which are anatomically associated with the site of the disease. $\mathrm{He}$ has observed them to be decidedly tender before they were in any degree enlarged or indurated. Often, indeed, if not commonly, the first change is rather a diminishing than an increase of their bulk, and a rounding of their form with bullety hardening. "I have met with very few instances in which the glands were not already affected, however recent the primary disease, and with still fewer in which they were not involved at the time of death." Upon the subject of treatment, Mr. Moore considers that the greatest benefit at present obtainable from medical treatment is that which is likely to arise from improving the general health; "as that fails, the disease advances; with its improvement the cancer-growth is stayed." Removal of the primary tumour by surgical operation lengthens life in some cases which were hastening to a fatal issue, and can rarely be accused of really shortening it. But, he remarks, "the majority of the cases selected for operation are such as present the least probability of early death; these give too favourable a view of the operation."

A short essay on Contusions, and a longer one on Wounds, conclude Mr. Paget's contributions to the volume. Mr. Paget describes the changes which take place in the parts contused, which may be studied in their proper textures, and in their bloodvessels and blood; and he also describes the mode in which reparation is effected after such injury. Upon the subject of incised wounds, Mr. Paget gives some good rules for their treatment, in which a large amount of practical observation is brought to bear on a subject on which writers have not often bestowed so much care. "There are comparatively few," he says, "in which the healing by immediate union is not desirable, or worth attempting."

The subject of Poisoned Wounds, or rather those received from poisons derived from the animal kingdom, is treated by Mr. Poland. The wounds so frequently received in dissection, as generally carried 
on, are not ordinarily attended with any peculiar or specific disease, or, we may add, with any characters which should separate them from other incised or punctured wounds. It is otherwise with the wounds -the "pricks" or "scratches"-received in the examination of recently dead bodies. More alarming symptoms follow these; in rare cases, it is true; but still they do happen with sufficient frequency to receive, as they require, a large amount of attention, and the more so, as they do also sometimes lead to a fatal result. These alarming symptoms, we think, arise more frequently in cases of inflamed serous membranes, especially the peritoneum.

After treating of these, Mr. Poland proceeds to consider separately the wounds received from healthy animals, the stings and bites of "venomous beasts," and those from diseased animals, of which hydrophobia and glanders are the best known and most frequent, and, we suppose we may add, with the general consent of our readers, the most uniformly fatal in their results in the human subject, as well as in the brutes from which they are derived.

In an essay by Mr. Moore, upon the general subject of Wounds of Bloodvessels, excluding those of the larger arteries and veins, which are to form the subject of other parts of the present series, he gives a good criticism upon the acknowledged modes of treatment in such cases. When giving in detail the rules applicable to ordinary hæmorrhage, Mr. Moore urges the importance, when bleeding continues, of clearing away the clots which have collected, and exposing the bleeding surface freely to the air. The effect of such treatment is sometimes exemplified in a remarkable degree when it becomes necessary to lay open the integument, and even the more deeply-seated parts. It seerns as if the blood, being injected into the areolar tissue, hinders the vessels from retracting efficiently within their sheaths. Many years ago, a man was brought into St. Bartholomew's Hospital, shortly after receiving a kick in the groin from a horse. A large and rapidlyincreasing swelling arose at the part. This was laid open freely by $\mathrm{Mr}$. Lawrence, the clots were cleared out, the hæmorrhage ceased, and no vessel required ligature. Free suppuration followed; and the wound eventually healed well.

The readiness with which arteries "escape threatened injury" is generally attributed to their great elasticity, or to the firmness of their outer coats. We think that some share of this freedom from danger may not unjustly be attributed to the nervous twigs from the sympathetic system which so abundantly surround the tubes. The large arteries have been known to escape injury when a bullet, or a piece of shell, has passed through a limb close to their natural course. A pitchfork has often been thrust through the neck immediately in front of the vertebræ without any bad consequence. In the riots in Paris in 1848, a cavalry soldier thrust his sword through a man's neck in this part, inflicting an injury from which the man recovered after a few days' rest in hospital.

Collapse, and the general effects of shock upon the system, are treated by Mr. Savory. To estimate the real effects, it seems to us 
that we must take "shock" in a vastly different number of cases. The effect will be different in the shock from an operation and in that from an injury; for one the patient is prepared, not so for the other. The effects will also be aggravated by loss of blood. Much obscurity still attends the cases of what are called "Death from Shock." Mr. Savory attributes the death from such a cause - the "purest example" of which is in a blow on the epigastrium-to a sudden and violent impression on some portion of the nervous system, acting at once through a nerve-centre upon the heart, and destroying its action," often producing death "without any detectable lesion." In these, as in some other cases, something more is required to be known beyond the facts, or absence of facts, discernible on a pathological examination, to enable us to say what was the real cause, physiologically speaking, of the fatal termination. The symptoms during life should be knownthe "history of the case," as we are in the habit of saying. The influence of respiration on disease, especially in its effects upon a severe surgical accident, has not been sufficiently noted. In the case of death from shock, what is the state of the lungs at the time of the injury? If slightly expanded, or, in common language, "empty," the little air is suddenly expelled by muscular contraction, and the patient dies; if full, emphysema follows. A carriage shall run away, and four ladies be thrown out as it is overturned; each will have her power of resistance to injury, in proportion as she has breath, or is without it.

Cases of death from "sudden and powerful emotions of the mind" are now and then reported in the medical journals. What happens when the patient does not die? Is recovery, as it is called, complete? Are no permanent traces left upon the system? A serjeant in a crack regiment of dragoons, on retiring from service, was appointed superintendent of a railway station. One day he attempted to pass his own child into the arms of the stoker of an engine which was passing the station; the child fell, and the truck following the engine went over it, causing immediate death. The father, up to that time a strong man, became so forgetful and inattentive as to be unfit for his duties. He was discharged and removed to a lunatic asylum; in a few months he died, from the general paralysis of the insane. The apparent cause of death was shown to be emphysema of the lungs, with congestion of the brain.

Mr. Holmes, the editor, contributes an essay on Burns and Scalds, and another upon the general pathology of dislocations. He follows Dupuytren's classification of burns and scalds, as being the most widely accepted, and the most complete and practical; observing that the exient of the burnt surface, as well as the depth to which the injury reaches, are important considerations, which, however eluding attempts at formal classification, should be taken into account in attempting a general arrangement, or one which shall embrace all the elements necessary for prognosis and treatment. "The extent of a scald often compensates, as it were, for the superficiality." In setting the pathology of these injuries before his readers, Mr. Holmes gives a valuable analysis, compiled from the case-books of St. George's Hospital, 
containing a full account of the history and post-mortem examinations of a large number of fatal cases. He thinks the causes and modes of death require to be more accurately determined, as they should serve for indications for the constitutional treatment of the patients. A short account is given of accidents from lightning-rare in this country, and of which few of us have seen many examples.

The general pathology and treatment of Fractures form the subject of the essay contributed by Mr. Hornidge, in which he has brought together within a short compass whatever is valuable in modern authors upon the subject he is treating.

The volume closes with a short essay by Mr. Holmes upon Dislocations, in which he discusses the general principles deducible from the pathology applicable to the treatment of traumatic dislocations; such displacements at least of one articular surface of bone from another as are caused by violence.

The general plan of the work, as far as we may be allowed to judge from the single volume before us, is well conceived, and the task has been executed with industry. The contributors have been well selected, and their united labour has been well expended. Mr. Holmes has done well in enlisting the services of the younger, though comparatively untried, writers, and writers from different schools. Without too much of what is merely historical, they have managed to place before the reader, in a small compass, and in clear essays, what is valuable in modern knowledge, and trustworthy as a guide in practice. The descriptions of diseases for the most part are good, and well drawn; and the symptoms well described. The frequent allusions in different essays to subjects not yet treated, show that the present volume is intended to be introductory to the fuller treatises which are to appear hereafter. From the ability displayed in the more excursive views appropriate as preliminary to a more extended subjectmatter, we shall look with well-grounded interest to the judicious concentration of practical details on special points. This we consider to constitute the essential core of a systematic abridgment of the present state of professional knowledge, furnishing at once easy access to condensed material, and the assistance of a discriminating experience. 